\title{
EL IMPACTO DE LA PROPIEDAD INSTITUCIONAL DE LAS ADMINISTRADORAS DE FONDOS DE PENSIÓN SOBRE LA POLÍTICA DE DIVIDENDOS: EVIDENCIA PARA CHILE*
}

Werner Kristjanpoller Rodríguez ${ }^{* *}$ Javiera Silva Arroyo ${ }^{* * *}$

doi:10.11144/Javeriana.cao27-49.ipia. Este artículo es producto de una investigación de los autores. El artículo se recibió el 07/07/2014 y se aprobó el 01/12/2014. Sugerencia de citación: Kristjanpoller R., W. y Silva A., J. (2014). El impacto de la propiedad institucional de las Administradoras de Fondos de Pensión sobre la política de dividendos: evidencia para Chile. Cuadernos de Administración, 27 (49), 11-36. http://dx.doi.org/10.11144/Javeriana.cao27-49. ipia

** Doctor en Ciencias Empresariales de la Universidad Autónoma de Madrid, Madrid, España, 2008. Profesor Jornada completa de la Universidad Técnica Federico Santa María, Valparaíso, Chile. Correo: werner.kristjanpoller@usm.cl

*** Magíster en Ciencias de la Ingeniería Industrial de la Universidad Técnica Federico Santa María, Valparaíso, Chile, 2014. Estudiante de Magíster de la Universidad Técnica Federico Santa María, Valparaíso, Chile. Correo electrónico: javiera.silva@alumnos.usm.cl 
El impacto de la propiedad institucional de las Administradoras de Fondos de Pensión sobre la política de dividendos: evidencia para Chile

\section{RESUMEN}

En este artículo se busca evidenciar el efecto del nivel de propiedad de las Administradoras de Fondos de Pensiones (AFP) chilenas y la política de dividendos de las empresas en las cuales invierten, mediante un análisis de datos de panel. Se encuentra evidencia de una relación positiva lineal y no lineal negativa entre el nivel de propiedad institucional de las AFP y la política de dividendos, representada por el rendimiento de los dividendos. Adicionalmente, se encuentra un sesgo de selección en esta relación, revelando que las AFP escogen empresas con mayores niveles de pago de dividendos, lo cual implica que las AFP se comportan como un inversor prudente y es apoyado por la hipótesis de pastoreo institucional.

Palabras clave: Administradoras de Fondos de Pensión, propiedad institucional, dividendos, Chile.

Clasificación JEL: G32, G35

The impact of
institutional ownership
of Pension Fund
Administrators on
dividend policy:
Evidence for Chile

The impact of of Pension Fund

Abstract

The goal of this paper is to determine the effect of the Chilean Pension Fund Administrator (PFA) ownership in to the dividend policy of the firms where they invest, through a panel data analysis. The results show a positive linear and no linear relationship between the PFA and the dividend policy, measured by the dividend yield. Also, it is found a selection bias in this relationship, indicating that PFA's prefer to invest in firms with higher dividends, implying that the AFP's behave as prudent investor and support the institutional herding hypothesis.

Keywords: Pension fund administrators, institutional ownership, dividends, Chile.

JEL Classification: G32, G35

\section{O impacto da} propriedade institucional das Administradoras de Fundo de Pensão sobre a política de dividendos: evidência para o Chile

\section{RESUMO}

Neste artigo, procura-se evidenciar o efeito do nível de propriedade das Administradoras de Fundo de Pensões (AFP) chilenas e a política de dividendos das empresas nas quais investem, mediante uma análise com dados em painel. Constata-se evidência de uma relação positiva linear e não linear negativa entre o nível de propriedade institucional das AFP e a política de dividendos, representada pelo rendimento destes. Além disso, constata-se uma tendência de seleção nessa relação, o que revela que as AFP escolhem empresas com melhores níveis de pagamento de dividendos, o que implica que as AFP se comportem como um investidor prudente e estejam apoiadas pela hipótese de pastoreio institucional.

Palavras-chave: Administradoras de fundo de pensão, propriedade institucional, dividendos, Chile.

Classificação JEL: G32, G35 


\section{Introducción}

Para comenzar es necesario declarar dos aspectos fundamentales: el primero es el impacto de los inversionistas institucionales sobre el comportamiento y las decisiones de las empresas. El segundo es la política de dividendos, la cual se considera como una de las decisiones relevantes dentro de las empresas, siendo definida por Black (1976) como un "rompecabezas". Tras reconocer la importancia de estos dos aspectos diversos estudios han analizado la relación entre la propiedad institucional y los dividendos, a saber Ruiz et al. (2008), Rubin y Smith (2009), Azzam (2010), entre otros.

La contribución de este artículo radica en la inclusión del caso chileno en la literatura, ampliando la visión en lo referente a países emergentes, los cuales no han tenido una presencia importante en las investigaciones. Las diferencias de estos países con respecto a las economías desarrolladas pueden conducir a resultados novedosos. Junto a esto, se considera que el contexto chileno presenta otros aspectos de interés para un posible estudio de la relación presentada: la importancia de las administradoras de fondos de pensiones: AFP (consideradas como participantes importantes y bien desarrollados dentro de la economía chilena, siendo un aspecto diferenciador en el grupo de mercados de capitales de países en desarrollo ${ }^{1}$ ) y el marco legal que

Las economías emergentes pueden tener alternativas de financiamiento más reducidas o limitadas en comparación con países desarrollados (Agosin y Pastén, 2003). Las economías desarrolladas poseen mercados de capitales complejos y amplios, por lo que las empresas en este contexto cuentas con diversas vías envuelve a las empresas; en relación al pago de dividendos por parte de las sociedades anónimas abiertas.

Se destaca que los inversionistas institucionales han contribuido a incrementar la profundidad, liquidez y eficiencia de su mercado (Gutiérrez et al., 2012), siendo las AFP los inversores más importantes por su contribución al desarrollo del mismo (Lefort y Walker, 2002) y han tenido una influencia notable en el proceso de toma decisiones corporativas de las empresas más reconocidas de Chile. Por otro lado, en esta economía sudamericana, las empresas operan en un marco legal que las obliga a pagar como mínimo un $30 \%$ de sus utilidades en forma de dividendos.

La inclusión del caso chileno encuentra respaldo en la posibilidad de que Latinoamérica represente diversas oportunidades de inversión a largo plazo para los inversionistas de varios países. Chile es un gran exponente dentro de esta región, siendo un importante país en vías de desarrollo que, en enero de 2010, pasó a ser el primer país sudamericano en incorporarse como miembro de la OCDE. En el Informe Mundial de Competitividad 2012, Chile se ubica en el lugar 28 de 59 países, ubicación que le permite mantener el liderazgo en la región y considerarse la economía más competitiva de Latinoamérica, todo esto gracias a su apertura comercial, estabilidad, fortaleza en el flujo de inversiones

de financiamiento. Luego, las AFP han reducido la cantidad de dinero prestado al sector público, hecho de gran importancia ya que implica que dichos fondos se destinan al sector privado, canalizando el capital disponible para las empresas (Finchelstein, 2012). 
foráneas, finanzas públicas, proyecciones de crecimiento, etc. Adicionalmente, Economist Intelligence Unit (EIU) ${ }^{2}$ señala que dichos factores han dado a Chile un ambiente de negocios atractivo y dinámico, lo que llevó a este país a ubicarse en el puesto número 20 entre las economías más llamativas para hacer negocios en el periodo 2010-2014, encabezando a las economías de la región.

Por estas razones el objetivo del presente artículo es analizar la relación entre la propiedad sobre las empresas que mantienen las AFP y los dividendos que estas empresas pagan, incluyendo diferentes variables de control, que permiten incorporar características de cada empresa en estudio, en la economía emergente chilena. Para cumplir el propósito de este estudio se utiliza una base de datos compuesta por 141 sociedades anónimas chilenas activas para un periodo de diez años comprendidos entre el 2003 y el 2012. Los datos financieros de las empresas fueron tomados de la base datos Economática (www.economatica.com), mientras que la información referente al nivel de participación de las AFP en las empresas se obtuvo de las bases de datos disponibles de la Superintendencia de Pensiones.

Los resultados obtenidos en este estudio evidencian la existencia de una relación positiva y significativa entre el rendimiento de los dividendos (calculado como el dividendo por acción sobre el precio de la misma) y el nivel de propiedad institucional de las AFP.

Unidad de negocios independiente dentro del grupo The Economist (www.eiu.com).
Este artículo se estructura de la siguiente manera: en la primera sección se presenta la revisión de la evidencia empírica que precede al estudio de este trabajo. La segunda sección presenta los modelos aplicados en el estudio, la definición de la muestra de estudio y las variables utilizadas. Seguido a esto, se muestran los resultados y su análisis. Finalmente, se exponen las conclusiones.

\section{Revisión de la literatura}

A lo largo del tiempo, la literatura ha reconocido la gran importancia de los inversores institucionales y su rol dentro del desarrollo de los mercados accionarios. Asimismo, dada su fuerte influencia dentro de las empresas, se reconoce el impacto que tienen estos inversores en el comportamiento de ellas y en sus decisiones; la razón de esto se relaciona con la capacidad de compra de grandes bloques de acciones y con la mayor información y conocimiento sobre las empresas comparado con el inversionista, aspectos que hacen que sus decisiones de inversión suelan tener mayor importancia dentro del mercado. Concretamente, se apunta a la idea de que una compra importante de acciones por parte de un inversor institucional puede ser vista como una señal positiva y, de manera similar, una venta de acciones puede ser una señal de desconfianza, por lo que la compra-venta de acciones de los inversores institucionales pueden tener un alto impacto en los precios de las acciones.

Revisando la evidencia empírica en relación al pago de dividendos y la participación institucional, se encuentran estudios como el de Howe et al. (1992), quienes realizaron un 
estudio basándose en dos tipos de transacciones: oferta de recompra de acciones y pagos de dividendos designados especialmente.

Allen et al. (2000) afirman que si en las empresas predominan los inversores bien informados (aquellos que mantienen relaciones de negocio con las empresas participadas, que tienen incentivos y capacidad para detectar la calidad de las empresas en las que ejercen su participación), se pagarán menos dividendos, ya que estos inversores pueden obtener beneficios privados a expensas de los no informados. En este contexto, investigaciones realizadas por Manos (2002) y Almazán et al. (2005), incorporan en su análisis la presencia de dichos inversionistas, planteando como hipótesis una relación positiva entre el ratio de pago de dividendos y la presencia de los inversionistas institucionales especialmente en ausencia de oportunidades de crecimiento. Maury y Pajuste (2002) estudian cómo diferentes estructuras de propiedad y diferentes tipos de dueños afectan las decisiones de pago de dividendos en Finlandia, encontrando que las firmas pagan mayores dividendos que cuando están controladas por inversores institucionales o corporaciones.

El estudio realizado por Short et al. (2002) examina tres modelos alternativos de dividendos y muestra una relación positiva entre la distribución de dividendos y la propiedad institucional en empresas pertenecientes al Reino Unido, lo que implicaría que para las empresas controladas por institucionales se espera un alto pago de dividendos.

Continuando con la revisión de la literatura referente a la relación lineal existente entre la propiedad institucional y la política de dividendos, se destaca el trabajo desarrollado por Rubin y Smith (2009), quienes encontraron que la política de dividendo tiene un impacto en la dirección de la relación entre propiedad institucional y volatilidad de la acción, es decir, el signo de la correlación entre propiedad institucional y volatilidad depende de la política de dividendos de la firma. La propiedad institucional está negativamente (positivamente) relacionada con la volatilidad entre acciones que no pagan (pagan) dividendos. Continuando la línea anterior, Azzam (2010) extiende el estudio de Rubin y Smith (2009) a Egipto, analizando el efecto de la propiedad institucional sobre la volatilidad de las acciones, los retornos y la política de dividendos y, de manera similar a la metodología utilizada por Rubin y Smith (2009), el impacto de la política de dividendos sobre la dirección de la relación entre la propiedad institucional y la volatilidad de las acciones y los retornos. Sus resultados muestran que, en esta economía, la propiedad institucional privada tiene un efecto negativo sobre el ratio de pago de dividendos.

Dado que este artículo se centra en la economía chilena, es necesario hacer una revisión de este contexto para examinar la relación entre la propiedad institucional y la política de dividendos. En primer lugar, se destaca que en Chile los inversionistas institucionales han contribuido en el desarrollo del mercado del país por medio de la participación de los fondos mutuos de renta fija y renta variable, los fondos de inversión, tanto nacionales como extranjeros $\mathrm{y}$, fundamentalmente, la participación de las administradoras de fondos de pensiones 
(AFP) (Gutiérrez et al., 2012), siendo estas últimas los inversores más importantes dentro del contexto chileno. El actual sistema de pensiones que rige en este país es consecuencia de reformas realizadas en la década de 1980 a partir del Decreto ley 3500 de noviembre de 1980, que entró en vigor el 1 de enero de 1983 (Agosín y Pastén, 2003). En este punto es importante destacar que, a causa de la privatización del sistema, los niveles de propiedad de estas instituciones aumentaron considerablemente, junto con su importancia dentro del mercado chileno, llegando a ser uno de los factores clave en su desarrollo (Lefort y Walter, 2002).

En países con poca protección de los inversores externos, como es el caso chileno, se observa una mayor concentración de propiedad, por lo que el principal conflicto de agencia se produce entre los accionistas controladores y los minoritarios (La Porta et al., 1999; Becht y Röell, 1999; Faccio et al., 2001; Gugler y Yurtoglu, 2003; Farinha y López de Foronda, 2005; Mancinelli y Ozkan, 2006). En estos contextos, los accionistas controladores son principalmente familias e inversores institucionales, los cuales deben ser considerados como agentes de gran importancia en el proceso de toma de decisiones, al tener los incentivos para influir de forma determinante en las mismas (La Porta et al., 1999; Faccio y Lang, 2002). En línea a lo anterior, Gugler y Yurtoglu (2003) afirman que los dividendos señalan la severidad del conflicto entre accionistas controladores y minoritarios. Así, los inversores institucionales influyen en las decisiones referentes a la política de dividendos de las empresas en las cuales ejercen su participación en función de sus preferencias.

Al ahondar en la política de dividendos en el caso chileno se debe considerar que en países desarrollados, como Estados Unidos, las empresas con grandes oportunidades de inversión son las que usualmente no pagan dividendos, porque las utilidades retenidas son la forma de financiamiento más conveniente a disposición de las empresas. Sin embargo, en Chile las empresas se ven obligadas a pagar dividendos y luego recaudar esos mismos fondos en el mercado de capitales, lo que supone una ineficiencia quizás innecesaria. Los dividendos en Chile son sometidos a un tratamiento menos ventajoso que el que afecta a las ganancias de capital, pero a pesar de eso, las empresas en Chile pagan dividendos ${ }^{3}$. Buena parte de esta aparente inconsistencia se relaciona con la regla del mandatory dividend que establece una obligación legal para las sociedades anónimas chilenas de pagar dividendos que representen al menos un 30\% de las utilidades líquidas. De acuerdo con La Porta et al. (2000) esta situación también se observa en otros países de Sudamérica.

En la revisión del estado del arte sobre el caso chileno, se destaca a Maquieira y Moncayo (2004) quienes presentaron un estudio (que replica el de Maquieira y Danús, 1998) que incluye la presencia de insiders, cuyos resultados muestran que en Chile los ratios

Las empresas en Chile distribuyen tres dividendos provisorios por año, más un dividendo mínimo obligatorio, que sólo se paga cuando los dividendos provisionales no alcanzan el mínimo para ser distribuidos (Nash y Fuenzalida, 2004). 
de pagos de dividendos tienen un contenido informacional (tasas de crecimiento esperadas mayores, implican mayores pagos de dividendos) y que una mayor propiedad accionaria de insiders se asocia a mayores pagos de dividendos. Fuenzalida et al. (2008) señalan que los inversionistas institucionales se preocupan más por el riesgo y la liquidez de sus inversiones que por establecer un ratio óptimo de dividendos.

La idea que la estructura corporativa en Chile se caracteriza por una alta concentración de propiedad y control (Lefort, 2007), es ratificada por el estudio de Gutiérrez et al. (2012) porque evidencian una relación directa entre la tasa de pago de dividendos y la concentración de la propiedad, potenciando la noción que la concentración de la propiedad implica un mayor pago de dividendos, lo cual sustentaría teóricamente el argumento de utilizar la política de dividendos para entregar información al mercado (pero no sobre proyectos futuros de la empresa, sino para indicar que los accionistas minoritarios son tratados en forma justa, supliendo de esta manera deficiencia del mercado chileno).

Ante los resultados anteriores presentados en los estudios motivados por la revisión de la relación en cuestión, se busca respaldo teórico que brinde soporte a la investigación. Con relación al pago de dividendos hay una serie de teorías sobre la preferencia de los inversionistas por un determinado nivel de pago de dividendos. Entre estos se destaca la planteada por Miller y Modigliani (1961), quienes mostraron que, en mercados competitivos y eficientes, la política de dividendos escogida por la empresa no tendrá un efecto en la riqueza de los accionistas de la empresa. Sin embargo, cuando se consideran imperfecciones en los mercados, esta irrelevancia de la política de dividendos puede desaparecer. Otros modelos basados en asimetrías de información (ligados a la señalización) suponen que los administradores de la firma poseen información sobre las oportunidades de inversión de la firma a la cual no tienen acceso los inversionistas; la relevancia no recae en el dividendo per se, si no en la información que los cambios en estos entregan. Se destacan también los modelos basados en costos de agencia, los cuales suponen un impacto positivo entre ambos aspectos, ya que el pago de dividendos es considerado como una forma de transmitir información sobre la empresa al mercado y de reducir la discrecionalidad directiva en el uso de los flujos de caja disponibles (Jensen, 1986), esto al tener que acudir a los mercados de capitales como fuente de financiación, lo que implica un mayor control (Rozeff, 1982). Esta teoría se sustenta en los problemas de agencia existentes entre los accionistas y los administradores; así, un aumento en el nivel de dividendos reduciría los flujos de caja libre a disposición de los administradores, disminuyendo el problema de agencia y aumentando el valor de la empresa. Por otro lado, existen teorías que indican que existe un menor riesgo asociado a un dólar de dividendos, comparado a un dólar obtenido mediante ganancias de capital, sugiriendo que los inversionistas preferirían compañías con mayores pagos de dividendos, siguiendo la idea de "pájaro en mano". También se encuentran teorías que supone que los dividendos están gravados con impuestos más altos que las ganancias de capital, por 
lo que los inversionistas se mostrarán con aversión a entrar en empresas que paguen altos dividendos.

En lo referente a los inversores institucionales, es necesario destacar ciertos aspectos importantes dentro de la teoría y que en este apartado serán revisados para el contexto chileno: la sofisticación institucional, la preferencia institucional y pastoreo institucional (Rubin y Smith, 2009; Azzam, 2010). El primero apunta a la idea de que los inversores institucionales están mejor informados que los otros inversores (Lin et al., 2007); los considerables recursos - dinero y capacidad de investigación- que invierten las instituciones hace que sea esperable que estas empresas tiendan a tener mucho más conocimiento e información que el inversor medio cuando se trata de las empresas e industrias en las que han invertido. De esta manera, es factible que sus decisiones de inversión suelan poseer gran peso para el resto de los inversores. La propiedad de las AFP puede afectar la política de dividendos debido a que ésta entrega un mensaje sobre el estado de la empresa y los inversores. El segundo aspecto apunta al comportamiento de las instituciones como un "inversor prudente"4. En este contexto, Fuentes (2013) estudia la hipótesis de "inversor prudente" para las AFP chilenas y, además, intentó determinar las características que más atraían a este tipo de inversores. Sus resultados muestran que las AFP se comportan como inversores pru-

Este comportamiento tiene su raíz en el hecho de que este tipo de inversores son fiduciarios (invierten dineros en nombre de otros), a diferencia de los inversores individuales. dentes, en parte, por la política regulatoria que las gobiernas y, en parte, por su propia gestión. Por último, el tercer aspecto se relaciona con la idea de que estos inversores tienden a moverse en "manada" (herding), respaldados por el comportamiento de otro par. En este aspecto, Sias (2004) documenta que los inversores institucionales siguen uno al otro en "manada" al momento de tomar determinadas decisiones. Además, Stein et al. (2011) miden el efecto de pastoreo (herding) en las posiciones de renta variable en Chile que mantienen las AFP, considerando el período 2003-2011. Sus resultados confirman la existencia de pastoreo en el mercado nacional, con intensidad moderada pero mayor que la reportada en los mercados accionarios de países desarrollados. Además, reportan que el pastoreo aparece con mayor fuerza en los momentos de crisis de los mercados, y tiende a desaparecer en períodos expansivos de la economía.

Dado que el estudio presentado incluye en los modelos características propias de las empresas que hacen el pago de los dividendos, se hace una revisión de la literatura relacionada con este aspecto. Pruitt y Gitman (1991) muestran que los beneficios actuales y pasados de la empresa son factores importantes que influyen en los pagos de dividendos. Por otro lado, Amihud y Murgia (1997) encuentran que una empresa tiene incentivos para favorecer el pago de dividendos estables inferiores y no para proteger su deuda de los riesgos de quiebra. En este sentido, los modelos teóricos sugieren que la deuda y los dividendos son dispositivos de sustitución para reducir los problemas de agencia o de la información asimétrica. Así, 
los flujos de caja libres (después de financiar todos los proyectos rentables) se pueden invertir inútilmente por los administradores que desean conservar el control sobre estos fondos. Ambas variables, deuda y el pago de dividendos, pueden reducir este problema al reducir la cantidad de flujo de caja libre.

Higgins (1972) muestra que los ratios de pago están negativamente relacionados con las necesidades de fondos de las empresas para financiar las oportunidades de crecimiento. Siguiendo la idea anterior, Higgins (1981) muestra la existencia de una relación directa entre el crecimiento de la firma y sus necesidades de financiamiento, por lo que sugiere que las empresas de rápido crecimiento poseen necesidades de financiamiento externo, esto debido a que las necesidades de capital circulante exceden normalmente los flujos de efectivo incrementales de las nuevas ventas. Intuitivamente, se espera que las empresas más grandes tengan pagos de dividendos más altos. Sin embargo, Barclay et al. (1995) muestran que las empresas de mayor tamaño poseen a su vez mayores responsabilidades, esto debido a que los tenedores de deuda tienen más confianza en las grandes empresas. Esto conduce a que las grandes empresas tiendan a pagar bajos dividendos a fin de no ampliar su endeudamiento.

Rozeff (1982) planteó que para empresas con oportunidades de crecimiento y alto riesgo es costoso recurrir al mercado, por lo cual, debieran pagar menos dividendos para ahorrar recursos.

Por último, se destaca que Ruiz et al. (2008) evidencian la existencia de una relación no lineal entre la propiedad institucional y los dividendos en el contexto español. Sus resultados muestran que existe un comportamiento heterogéneo de los distintos tipos de entidades analizadas en relación con la política de dividendos de las empresas participadas. El signo de la relación dependería de la naturaleza del inversor institucional. Esto motiva la revisión de esta relación no lineal para el caso chileno.

\section{Metodología y data}

Este estudio se basa en explicar la influencia de la propiedad que mantienen las AFP en las empresas chilenas, principalmente en lo relacionado con la política de dividendos. Para ello se utiliza como proxy el rendimiento de los dividendos (DIVY) como variable dependiente, medida que ha sido utilizada en estudios como el de Smith y Watts (1992) y que se calcula como el dividendo de los últimos 12 meses sobre el precio de la acción.

Esta medida es un indicador de la rentabilidad esperada para el mercado bursátil, lo que motiva su utilización dado que contiene información de mayor interés para los inversionistas en relación con el rendimiento del dividendo recibido por sus inversiones y a que esta medida tiene un gran poder predictivo en el mercado chileno (Lefort y Walker, 2002). La utilización del rendimiento del dividendo tiene por objetivo analizar una variable que incorpora la valorización por parte del mercado, lo cual implica que se tiene en cuenta las proyecciones de flujos de caja futuro y riesgo asignado al usar como base del ratio el precio de mercado. Por otro lado, la variable independiente de 
este estudio representa el nivel de propiedad institucional de las AFP (PAFP) presente en las empresas chilenas en estudio. Esta medida representa el porcentaje de acciones en poder de estas instituciones sobre el total de acciones emitidas por una empresa $i$ en un periodo $t$.

De acuerdo con varios autores, como Maquieira y Fuentes (1997), Maquieira y Danus (1998), Maquieira y Moncayo (2004), Ruiz et al. (2008) y Azzam (2010), entre otros, se sugiere que el pago de dividendos de las firmas puede relacionarse con diferentes variables financieras y características de la precio de mercado y el precio valor libro de la acción $(P / V L)$, lo que representa la valorización o desvalorización de la acción de la firma. Por último, se considera el índice $\mathrm{Q}$ de Tobin $(\mathrm{Q})$ como proxy del flujo de caja libre (FLUJOS), calculado como la suma entre pasivos y capitalización bursátil sobre el total de activos, y la señalización de las ganancias $(S E N)$ calculado como la diferencia entre la utilidad por acción en el periodo t y la utilidad por acción en $\mathrm{t}-1$ sobre el precio de la acción en el periodo t-1 (SEN) (Maquieira y Fuentes, 1997). El modelo se representa en la ecuación (1).

$$
\begin{aligned}
& D_{V I V} Y_{i t}=\alpha_{i t}+\beta_{p a t p} \cdot P A F P_{i t-1}+\beta_{\text {tam }} T A M_{i t}+\beta_{\text {apal }} \cdot \operatorname{APALAN}_{i t-1}+\beta_{\text {rent }} \cdot \operatorname{RENT}_{i t}+\beta_{\text {opor }} . \\
& \operatorname{OPOR}_{i t}+\beta_{\text {flujo }} \cdot F L U J O S_{i t-1}+\beta_{\text {sen }} \cdot \operatorname{SEN}_{i t}
\end{aligned}
$$

firma, por lo que es necesario controlarlas en los modelos tales como el tamaño (TAM) de la empresa, empleando dos diferentes medidas: el logaritmo de los ingresos netos (TAM1) y el logaritmo de la capitalización bursátil (TAM2). También incluimos variables para el control del rendimiento contable (RENT) utilizando la rentabilidad sobre el patrimonio (ROE) y la rentabilidad sobre los activos (ROA). Además, se incluyen los ratios de deuda total sobre el valor libro de los activos totales (APAL1) y deuda total sobre el valor libro del patrimonio (APAL2) para controlar el apalancamiento (APALAN). Por otro lado, las oportunidades de crecimiento $(O P O R)$ de la firma (OPCRE) se caracterizan mediante la inclusión del ratio ${ }^{5}$ entre

Un alto nivel de este ratio puede asociarse a la expectativa de los flujos futuros que puede llegar a recibir
Se considera el rezago en un periodo respecto a la variable dependiente para las medidas de propiedad institucional de las AFP (PA$\mathrm{FP})$, apalancamiento $(A P A L A N)$ y para la $\mathrm{Q}$ de Tobin (Q), con el fin de evitar posibles problemas de endogeneidad (Giner y Salas, 1995; Grinstein y Michaely, 2005; Ruiz et al., 2008). Mediante la metodología de datos de panel se generan las distintas estimaciones a partir de la ecuación (1), utilizando la prueba de Hausman para determinar la conveniencia entre utilizar efectos fijos o aleatorios.

Dado que existen empresas que no pagan dividendos a pesar de la obligatoriedad del

la firma, mientras un bajo valor puede deberse a una mala administración de los recursos observada por el mercado. 
marco legal chileno, sea por no haber generado utilidades durante el periodo o por de- drática, lo que permitiría la estimación no lineal de dicha relación, según ecuación 2.

$$
\begin{aligned}
& D I V Y_{i t}=\alpha_{i t}+\beta_{\text {pafp }} \cdot P A F P_{i t-1}+\beta_{\text {pafp } 2} \cdot P A F P_{i t-1}^{2}+\beta_{\text {tam }} \cdot \operatorname{TAM}_{i t}+\beta_{\text {apal }} \cdot A P A L A N_{i t-1}+ \\
& \beta_{\text {rent }} \cdot \operatorname{RENT}_{i t}+\beta_{\text {opor }} \cdot \operatorname{OPOR}_{i t}+\beta_{\text {flujo }} \cdot \operatorname{FLUJO}_{i t-1}+\beta_{\text {sen }} \cdot \operatorname{SEN}_{i t}
\end{aligned}
$$

cidir pagar dividendos atrasados, la variable dependiente puede ser considerada como una variable censurada. Muchos estudios precedentes con relación a los determinantes de los dividendos utilizan el modelo Tobit, el cual logra controlar la existencia de ceros en el proceso de estimación (Arrazola et al., 1992; Giner y Salas, 1995; Han et al., 1999; Maury y Pajuste, 2002; Gugler y Yurtoglu, 2003; Farinha y López de Foronda, 2005; Mancinelli y Ozkan, 2006; Ruiz et al., 2008). Esto motiva a utilizar el modelo de Tobit de panel sobre el modelo (1) presentado anteriormente ${ }^{6}$.

Chen et al. (2004) demuestran la importancia de las relaciones no lineales en la investigación en finanzas corporativas y destacan que las especificaciones no lineales en los modelos de regresión han ganado atención en las investigaciones en el ámbito de las finanzas corporativas, y en particular en los estudios sobre estructura de propiedad. Esto motiva la inclusión del estudio de la relación entre los dividendos y el nivel de propiedad de las AFP incluyendo el modelo (2) que contiene la variable explicativa PAFP en forma cua-

El modelo de Tobit de panel logra controlar la heterogeneidad no observable causada por la peculiaridad propia de las empresas, lo que implica una ventaja frente al análisis de corte transversal.
Finalmente, se analiza la presencia de sesgo de selección en el modelo propuesto. En este aspecto se plantea la posibilidad de que las AFP escojan empresas con un cierto nivel de pago de dividendos y que estos, una vez siendo propietarios de la empresa de interés, impacte en los pagos. Para estudiar esto, se utiliza el modelo de Heckman de dos etapas. En su realización se generaron dos variables auxiliares DIVYSI (la cual toma el valor $1 \mathrm{si}$ el rendimiento de los dividendos es mayor a cero y 0 en el caso contrario) y PAFPSI (la cual toma el valor 1 si la empresa ejerce una propiedad mayor a cero y 0 en el caso contrario).

Para este estudio se utiliza una muestra que incluye 141 sociedades anónimas abiertas chilenas entre los años 2003 y 2012, cuyos datos financieros fueron obtenidos de la base de datos de Economática ${ }^{7}$. La información de los porcentajes referentes al nivel de propiedad institucional de las AFP fueron obtenidos de las bases datos disponibles de la Superintendencia de Pensiones (www. spensiones.cl).

Teniendo un promedio de 1280 observaciones en nuestra muestra (con un máximo de 1486 observaciones para PAFP y un mínimo

Los datos financieros reportados por esta fuente están consolidados en moneda local. 
de 1088 observaciones para SEN), en el cuadro 1 se observa que las empresas presentan en promedio una rentabilidad del dividendo de $3,96 \%$, lo cual puede considerarse como moderado. Por otro lado la propiedad promedio de los fondos de pensiones (PAFP) para las empresas consideradas en nuestra muestra alcanza un $4,1 \%$, teniendo como máximo un $27 \%$ de propiedad sobre el total de acciones de una empresa determinada en un tiempo dado. En cuanto a las medidas de tamaño (TAM1 y TAM2), se aprecia que estas presentan un promedio similar entre ellas (aproximadamente con un valor de 19), al igual que sus rangos máximos y mínimos, por lo que es posible establecer cierta similitud entre estas variables y consistencia en cuanto al control del tamaño de la empresa. Por otro lado, en lo referente a las medidas de apalancamiento (APAL1 y APAL2), se observan diferencias en valores promedios (45,38\% y $175,76 \%$ respectivamente) y en los rangos de valores que toman, por lo que muestran distintas magnitudes de apalancamiento para las empresas. Para las medidas de rentabilidad contable, se observan promedios de 5,57\% y 9,5\% para las variables ROA y ROE respectivamente. En el cuadro 1 se relacionan las estadísticas descriptivas para las variables de estudio.

El cuadro 2 muestra las correlaciones entre las variables de estudio. Se destacan la alta correlación para las medidas de rentabilidad contable ROA y ROE $(0,8222)$; las medidas de tamaño para la firma presentan altas correlaciones entre sí. Observando las medidas de apalancamiento, se destaca

Cuadro 1. Sociedades anónimas chilenas, 2003-2012

\begin{tabular}{|c|c|c|c|c|c|}
\hline \multirow{2}{*}{ Variable } & \multirow{2}{*}{ Observaciones } & \multirow{2}{*}{ Medias } & \multirow{2}{*}{ Desviación estándar } & \multicolumn{2}{|c|}{ Valores límite } \\
\hline & & & & Min & Max \\
\hline DIVY & 1.436 & 0,0396 & 0,0809 & 0,0000 & 0,9810 \\
\hline PAFP & 1.486 & 0,0416 & 0,0645 & 0,0000 & 0,2708 \\
\hline TAM1 & 1.122 & 18,6832 & 2,1153 & 7,3976 & 23,1229 \\
\hline TAM2 & 1.340 & 19,0872 & 1,9119 & 13,4799 & 23,2602 \\
\hline APAL1 & 1.255 & 45,3818 & 21,1418 & 0,0000 & 94,4000 \\
\hline APAL2 & 1.255 & 175,7589 & 274,9544 & 0,0000 & 1678,3540 \\
\hline SEN & 1.088 & 0,0473 & 10,8300 & $-312,4886$ & 134,9063 \\
\hline Q & 1.170 & 46,8185 & 20,7195 & 0,8095 & 110,0105 \\
\hline OPCRE & 1.191 & 2,3359 & 6,5632 & 0,1000 & 111,3000 \\
\hline ROA & 1.367 & 5,56949 & 11,0855 & $-101,3523$ & 92,1557 \\
\hline ROE & 1.250 & 9,4979 & 20,7718 & $-206,8000$ & 205,8000 \\
\hline
\end{tabular}

Nota: la muestra considera los datos de 141 sociedades anónimas chilenas.

Fuente: elaboración propia. 
Cuadro 2. Correlaciones entre cada una de las variables

\begin{tabular}{|l|c|c|c|c|c|c|c|c|c|c|c|}
\hline & DIVY & PAFP & TAM1 & TAM2 & APAL1 & APAL2 & Q & OPCRE & SEN & ROA & ROE \\
\hline DIVY & 1,0000 & & & & & & & & & & \\
\hline PAFP & 0,0570 & 1,0000 & & & & & & & & & \\
\hline TAM1 & $-0,0094$ & 0,3412 & 1,0000 & & & & & & & & \\
\hline TAM2 & 0,0159 & 0,3361 & $0,7426^{*}$ & 1,0000 & & & & & & & \\
\hline APAL1 & $-0,0545$ & 0,1058 & 0,3969 & 0,0740 & 1,0000 & & & & & & \\
\hline APAL2 & $-0,0813$ & $-0,0606$ & 0,2151 & $-0,0088$ & $0,7149^{*}$ & 1,0000 & & & & & \\
\hline Q & $-0,0488$ & 0,1057 & 0,3973 & 0,0882 & $0,9985^{*}$ & $0,7083^{*}$ & 1,0000 & & & & \\
\hline OPCRE & 0,0431 & 0,0136 & 0,1348 & 0,3632 & 0,1886 & 0,0562 & 0,2289 & 1,0000 & & & \\
\hline SEN & 0,0638 & $-0,0087$ & $-0,0137$ & $-0,0200$ & 0,0318 & 0,0236 & 0,0317 & 0,0100 & 1,0000 & & \\
\hline ROA & 0,2076 & $-0,0182$ & 0,1404 & 0,2904 & $-0,1087$ & $-0,1593$ & $-0,0883$ & 0,3874 & 0,1767 & 1,0000 & \\
\hline ROE & 0,1968 & $-0,0104$ & 0,1343 & 0,2760 & $-0,0193$ & $-0,0754$ & 0,0001 & 0,4879 & 0,1400 & $0,8244^{*}$ & 1,0000 \\
\hline
\end{tabular}

* Correlaciones considerablemente altas.

Fuente: elaboración propia.

una alta correlación entre APAL1 y APAL2 $(0,7149)$. En estos casos, estas correlaciones no afectan los modelos propuestos, ya que no son incluidas en el modelo de manera simultánea, sino que se incluyen de manera separada para controlar determinadas características de las firmas. Además, se destacan las correlaciones entre la Q de Tobin (Q) y las medidas de apalancamiento APAL1 y APAL2 $(0,9985$ y 0,7080 respectivamente), lo cual debe ser considerado en el análisis de los modelos generados para este estudio.

\section{Análisis de resultados}

Utilizando datos de panel, se generaron 18 regresiones a partir del modelo (1), resultantes de las diferentes combinaciones de las medidas utilizadas para controlar cada una de las características de las firmas consideradas en este estudio. Todas las estimaciones tienen en común las variables de control $O P C R E$ y $\mathrm{Q}$, variando en las medidas de tamaño (TAM), apalancamiento (APALAN) y rentabilidad contable (RENT). Se destaca que debido a la alta correlación existente entre las medidas de apalancamiento APAL1 y APAL2 con la variable $Q$, las regresiones son generadas sin incluir estas variables de manera simultánea, lo que hace no previsible que se presenten problemas de multicolinealidad en las estimaciones.

Los cuadros 3 y 4 presentan los resultados de estas regresiones. Seguido a esto, la prueba de Hausman determina la conveniencia entre incluir en cada regresión efectos fijos o aleatorios y los resultados arrojados muestran que para todas las estimaciones generadas 
en este estudio puede realizarse la inclusión tanto de efectos aleatorios como de efectos fijos, optándose por estos últimos.

El principal resultado muestra que, para todas las regresiones, el nivel de propiedad institucional tiene un efecto positivo y significativo (al 95\% controlando TAM1 y 99\% controlando TAM2) sobre la política de dividendos, representada por el rendimiento de los dividendos. Estos resultados difieren, en parte, a los encontrados por Azzam (2010), quien señala que la propiedad institucional tiene un impacto negativo en el pago de dividendos, y por Allen et al. (2000) quienes afirman que si en las empresas predominan los inversores bien informados (entre los cuales incluimos a los inversores institucionales, ya que estos tienen incentivos y capacidad para detectar la calidad de las empresas en las que ejercen su participación) se pagarán menos dividendos, pero son respaldados por los expuestos por Short et al. (2002) para el caso de Reino Unido; Manos (2002), Almazán et al. (2005), Maury y Pajuste (2002), quienes también encuentran una relación positiva entre los pagos de dividendos y la propiedad de institucionales.

En cuanto a las variables de control incluidas en las estimaciones se destaca la relación positiva entre la variable dependiente y ambas medidas de rentabilidad contable (ROA y ROE), mostrando un alto nivel de significancia (99\%) en las 12 regresiones obtenidas. Esto puede evidenciar que rentabilidades mayores indican una mayor capacidad de la firma para pagar mayores dividendos. En cuanto al tamaño de la empresa se observa una relación negativa y significativa en todas las regresiones que controlan TAM2 y para la mayoría de las regresiones que controlan para TAM1, resultados que respaldan lo planteado por Barclay et al. (1995) respecto a que las empresas de mayor tamaño poseen a su vez mayores responsabilidades, conduciendo a estas firmas a pagar bajos dividendos a fin de no endeudarse aún más. Por último, en las estimaciones (3), (6), (9) y (12) se observa una relación positiva (a un nivel de significancia del 90\%) entre el rendimiento de los dividendos y la señalización de las ganancias, lo cual puede indicar que las empresas incrementan sus dividendos únicamente cuando esperan que sus ganancias aumenten (Maquieira y Moncayo, 2004).

El siguiente paso es la estimación de las regresiones para el modelo (1) utilizando el método de Tobit. Los resultados de esto se presentan en el cuadro 4. Los resultados expuestos muestran una relación positiva; con una significancia del $99 \%$ en la gran mayoría de las estimaciones, salvo en las (14) y (19) las cuales alcanzan un nivel del $95 \%$, lo que implica que un aumento en la propiedad institucional de las AFP durante un periodo dado (traducido en un aumento de la variable PAFP) aumenta la probabilidad de que el rendimiento de los dividendos (DIVY) pase a niveles más altos en el siguiente periodo.

Ahondando en el análisis de la relación estudiada, se estima mediante el procedimiento de Tobit, el modelo (2) para determinar la existencia de una relación no lineal entre las variables de estudio. Los resultados de las estimaciones realizadas en esta etapa del estudio se detallan en el cuadro 5. 


\begin{tabular}{|c|c|c|c|c|c|c|c|c|c|c|c|c|c|c|}
\hline \multirow{13}{*}{ 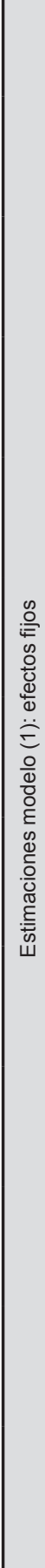 } & $\stackrel{ }{\sim}$ & 离 & & 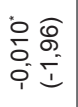 & & $\begin{array}{l}8 \widehat{\hat{6}} \\
80 \\
0.0\end{array}$ & $\begin{array}{l}8 \widehat{0} \\
00 \\
i 0\end{array}$ & $\begin{array}{l}\infty \\
0 \tilde{0} \\
0= \\
0=\end{array}$ & & 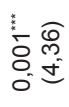 & & $\begin{array}{l}\stackrel{*}{\mathbb{N}} \\
\underset{\sim}{N} \\
0\end{array}$ & 음 & 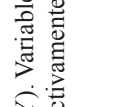 \\
\hline & $\mp$ & 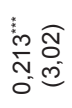 & & 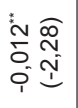 & & 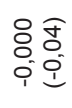 & 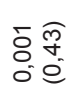 & $\begin{array}{l}8 \underset{\text { N }}{0} \\
0=\end{array}$ & ڤั & & & 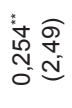 & ஜூ & 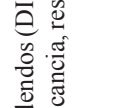 \\
\hline & 음 & कू⿻ & & 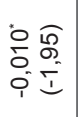 & 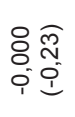 & & $\begin{array}{l}8 \widehat{0} \\
0.0 \\
01\end{array}$ & 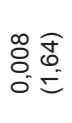 & & 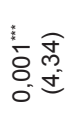 & & $\begin{array}{l}\stackrel{\text { }}{\mathbb{N}} \\
\underset{0}{\mathbb{N}} \\
0\end{array}$ & 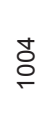 & 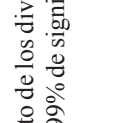 \\
\hline & $\sigma$ & 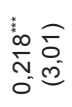 & & 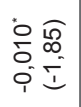 & & & $\begin{array}{l}\text { 응 } \\
0.0 \\
i=1\end{array}$ & $\begin{array}{l}\dot{0} 0 \\
00 \\
0= \\
0=\end{array}$ & & 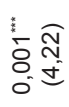 & 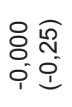 & 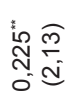 & ஜ্চু & 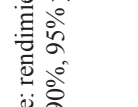 \\
\hline & $\infty$ & 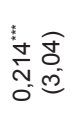 & & 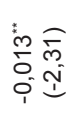 & 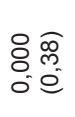 & & $\begin{array}{l}\bar{\delta} \underset{\mathfrak{g}}{\stackrel{9}{0}} \\
0\end{array}$ & 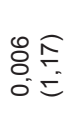 & 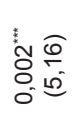 & & & 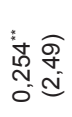 & ஜூ & 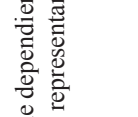 \\
\hline & $\wedge$ & 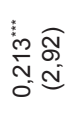 & & 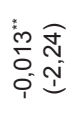 & & & \begin{tabular}{l}
$\bar{\delta}$ \\
$\bar{m}$ \\
0 \\
\hdashline
\end{tabular} & 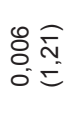 & 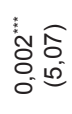 & & 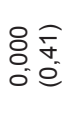 & 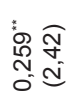 & Б్ & $\stackrel{i}{0}^{*}$ *. \\
\hline & 0 & 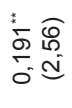 & 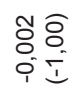 & & & 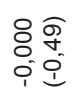 & 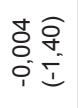 & 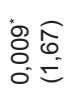 & & $\begin{array}{l}1 \\
\overline{8} \\
0 \\
0 \\
0\end{array}$ & & $\begin{array}{l}0 \widehat{0} \\
0: 0 \\
0= \\
0=\end{array}$ & চ্் & 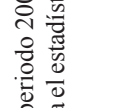 \\
\hline & 10 & 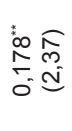 & $\begin{array}{l}\text { in } \widehat{n} \\
0.5 \\
0=\end{array}$ & & & 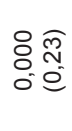 & 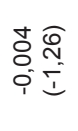 & $\begin{array}{l}8 \stackrel{6}{\frac{6}{2}} \\
0 \\
=\end{array}$ & 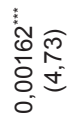 & & & 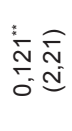 & ஜ & 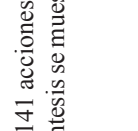 \\
\hline & $\nabla$ & 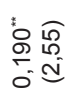 & $\begin{array}{l}\text { mo } \\
80 \\
0.5 \\
0=\end{array}$ & & 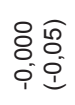 & & 亲 & $\begin{array}{l}8 \longdiv { 0 } \\
80 \\
0=\end{array}$ & & 象 & & 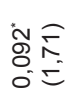 & চ্் & 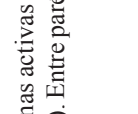 \\
\hline & $m$ & $\begin{array}{l}\infty \\
\infty \\
\infty \\
\infty \\
0 \\
0 \\
0\end{array}$ & $\begin{array}{l}\text { mo } \\
80 \\
15\end{array}$ & & & & 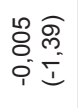 & 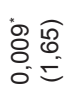 & & $\begin{array}{l}\frac{1}{\rho} \\
\stackrel{0}{0} \\
0 \\
0\end{array}$ & $\begin{array}{l}\text { 응으 } \\
\text { 웅 }\end{array}$ & 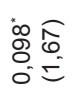 & $\begin{array}{l}\mathscr{E} \\
\varnothing\end{array}$ & 吾 \\
\hline & $\sim$ & 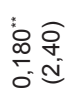 & 造 & & $\begin{array}{l}8 \overline{0} \\
0.0 \\
0\end{array}$ & & 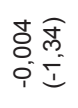 & 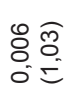 & $\begin{array}{l}\vdots \\
\vdots \\
\delta^{\circ} \\
0 \\
0 \\
0\end{array}$ & & & 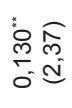 & ஜ & 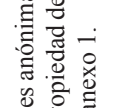 \\
\hline & - & 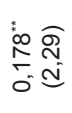 & 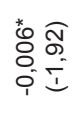 & & & & 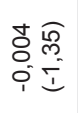 & 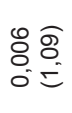 & 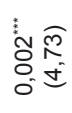 & & $\begin{array}{l}8 \widehat{\circ} \\
000 \\
00\end{array}$ & 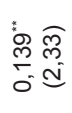 & $\begin{array}{l}\infty \\
\stackrel{\infty}{\infty}\end{array}$ & 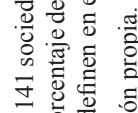 \\
\hline & $\begin{array}{l}\frac{0}{0} \\
\frac{0}{\frac{\pi}{2}} \\
\frac{10}{5}\end{array}$ & $\underset{\frac{0}{4}}{\Delta}$ & $\sum_{i}^{\Sigma}$ & $\sum_{\leqslant}^{N}$ & $\frac{\bar{\alpha}}{\frac{1}{\alpha}}$ & $\frac{N}{\frac{1}{\alpha}}$ & $\begin{array}{l}\text { 崩 } \\
0 \\
0 \\
0\end{array}$ & 岕 & \ీ & $\begin{array}{l}\text { 山् } \\
\text { ه }\end{array}$ & ○ & $\stackrel{⿱ 山}{\mathrm{U}}$ & $z$ & 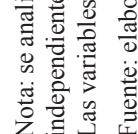 \\
\hline
\end{tabular}




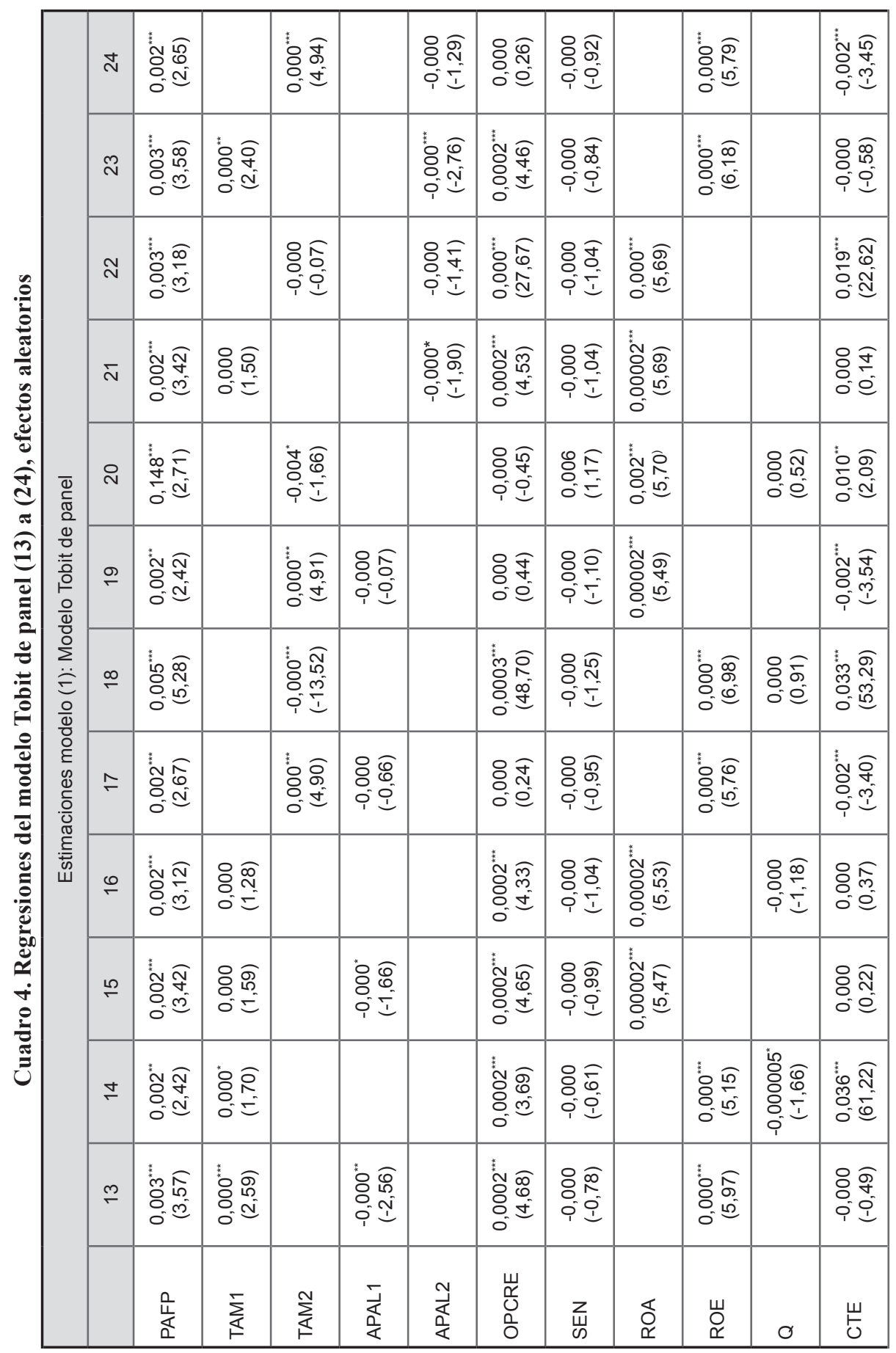




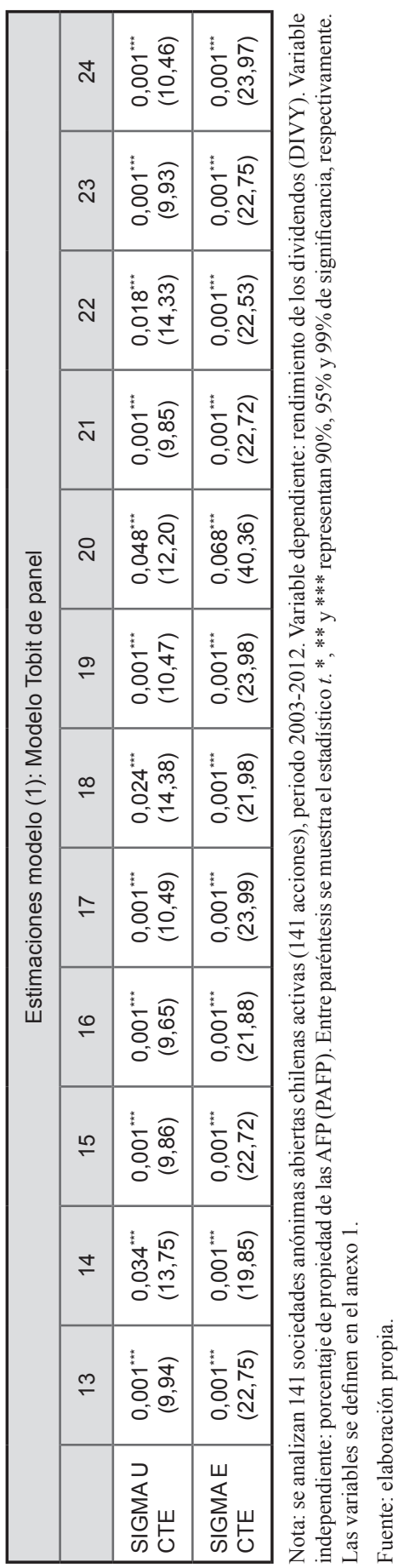

\begin{tabular}{|c|c|c|c|c|c|c|c|}
\hline \multirow{13}{*}{ 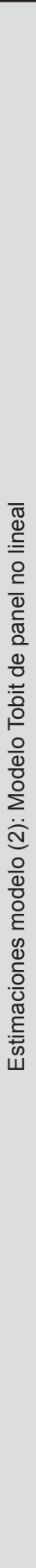 } & $\stackrel{m}{\infty}$ & 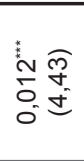 & $\begin{array}{l}0 \\
0 \\
0 \\
0 \\
0 \\
0 \\
0 \\
1 \\
1\end{array}$ & & 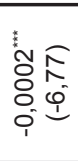 & & 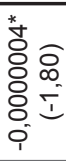 \\
\hline & $\stackrel{m}{m}$ & 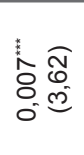 & 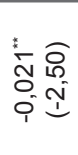 & 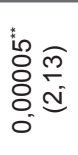 & & & 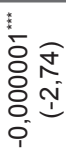 \\
\hline & ఉా & 蛋 & 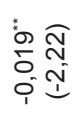 & & 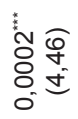 & & 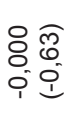 \\
\hline & ल्ల & 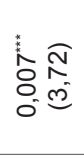 & 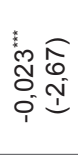 & $\begin{array}{l}8 \widehat{\circ} \\
0.0 \\
0=\end{array}$ & & & 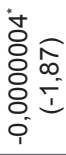 \\
\hline & ల్ & 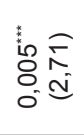 & $\begin{array}{l}0 \\
\infty \\
0 \\
0 \\
0 \\
0 \\
0 \\
0\end{array}$ & & 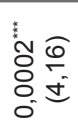 & & \\
\hline & $\bar{m}$ & 焉 & 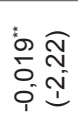 & & 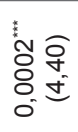 & $\begin{array}{l}80 \\
80 \\
0 \\
1\end{array}$ & \\
\hline & ஓ & $\begin{array}{l}\hat{\jmath} \\
\hat{0} \\
0 \\
0 \\
0\end{array}$ & 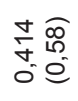 & & 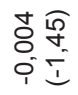 & & \\
\hline & D & 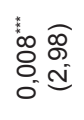 & 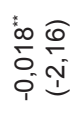 & & 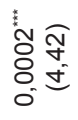 & $\begin{array}{l}80 \\
80 \\
0 \\
1 \\
1\end{array}$ & \\
\hline & $\stackrel{\sim}{\sim}$ & $\begin{array}{l}\text { : } \\
0 \\
0 \\
0 \\
0\end{array}$ & 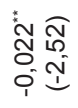 & $\begin{array}{l}\text { 응 } \\
0= \\
0=\end{array}$ & & & \\
\hline & $\hat{N}$ & 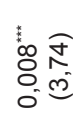 & 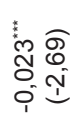 & $\begin{array}{l}\text { ৪ } \\
\text { 心 } \\
0=\end{array}$ & & 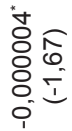 & \\
\hline & $\stackrel{\leftrightarrow}{\sim}$ & 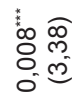 & 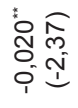 & 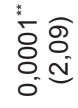 & & & \\
\hline & $\stackrel{2}{\sim}$ & 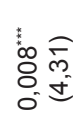 & 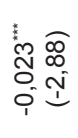 & 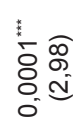 & & 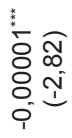 & \\
\hline & & $\underset{\frac{0}{4}}{\underline{\alpha}}$ & 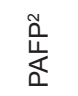 & $\sum_{i}^{\Sigma}$ & $\sum_{i}^{N}$ & $\frac{\bar{c}}{\frac{1}{\alpha}}$ & $\frac{\text { Tे }}{\frac{1}{\alpha}}$ \\
\hline
\end{tabular}




\begin{tabular}{|c|c|c|c|c|c|c|c|c|c|c|}
\hline \multirow{13}{*}{ 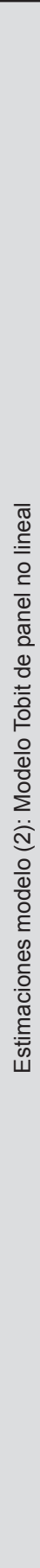 } & ஜ్ల & 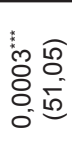 & 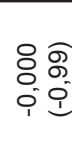 & & 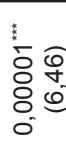 & & $\begin{array}{l}0 \\
0 \\
0 \\
0 \\
0 \\
0 \\
0\end{array}$ & $\begin{array}{l}\widehat{N} \\
\stackrel{\sim}{\sim} \\
0 \\
0 \\
0\end{array}$ & 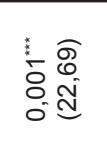 & \multirow[b]{13}{*}{ 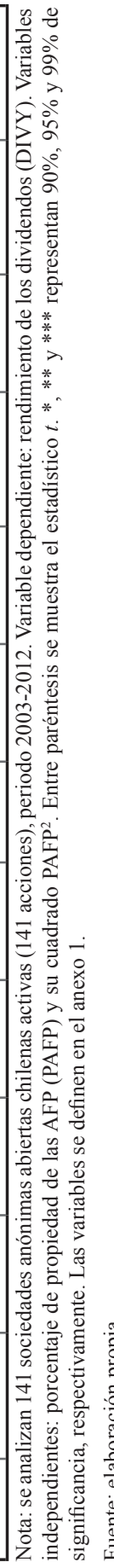 } \\
\hline & 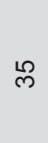 & $\begin{array}{l}\frac{1}{0} \\
\delta \\
0 \\
0 \\
0\end{array}$ & $\begin{array}{l}8 \\
8 \\
0 \\
0 \\
0 \\
\\
\end{array}$ & & $\begin{array}{l}1 \\
\delta \\
8 \\
0 \\
0\end{array}$ & & 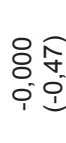 & 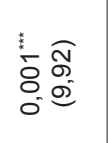 & 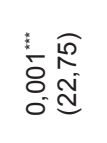 & \\
\hline & ষे & 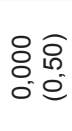 & 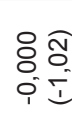 & 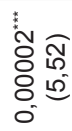 & & & 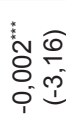 & $\begin{array}{l}1 \\
\overline{8} \\
8 \\
0 \\
0 \\
0\end{array}$ & $\begin{array}{l}1 \\
\overline{8} \\
\overline{8} \\
0 \\
0 \\
0\end{array}$ & \\
\hline & ల్ల & 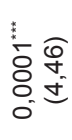 & $\begin{array}{l}8 \text { 于 } \\
80 \\
0 \\
\\
\end{array}$ & 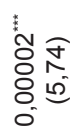 & & & 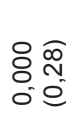 & $\begin{array}{l}1 \\
\delta \\
8 \\
0 \\
0 \\
0\end{array}$ & 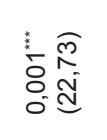 & \\
\hline & ల్లె & 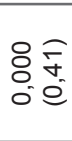 & 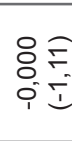 & 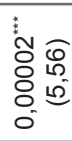 & & 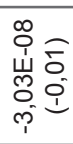 & 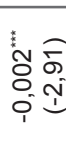 & $\begin{array}{l}5 \widehat{N} \\
8 \\
0 \\
0 \\
0\end{array}$ & 产 & \\
\hline & $\bar{m}$ & $\begin{array}{l}80 \\
80 \\
0.00 \\
0.0\end{array}$ & $\begin{array}{l}8 \widehat{0} \\
85 \\
0 \\
\end{array}$ & 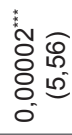 & & & స̃ & $\begin{array}{l}\hat{y} \\
8 \\
8 \\
0 \\
0 \\
0\end{array}$ & 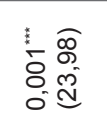 & \\
\hline & ిల & 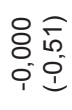 & 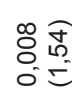 & & $\begin{array}{l}1 \\
\overline{0} \\
0 \\
0\end{array}$ & 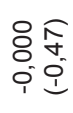 & 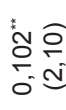 & 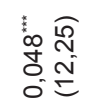 & 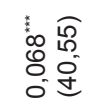 & \\
\hline & D & 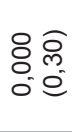 & $\begin{array}{l}8 \overline{0} \\
80 \\
0 \\
\end{array}$ & & 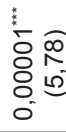 & & 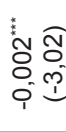 & $\begin{array}{l}1 \\
\overline{0} \\
8 \\
0 \\
0\end{array}$ & 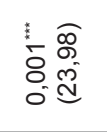 & \\
\hline & $\stackrel{\infty}{\sim}$ & 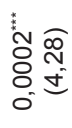 & 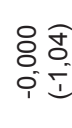 & 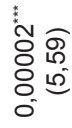 & & $\begin{array}{l}80 \\
8 \\
0 \\
0\end{array}$ & $\begin{array}{l}80 \\
80 \\
00 \\
00\end{array}$ & $\begin{array}{l}\overline{8} \\
80 \\
0 \\
0\end{array}$ & 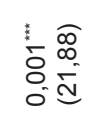 & \\
\hline & $\hat{\sim}$ & 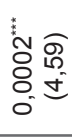 & 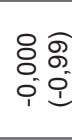 & 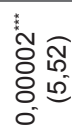 & & & 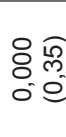 & $\begin{array}{l}\overline{1} \\
\bar{\delta} \\
0 \\
0 \\
0\end{array}$ & 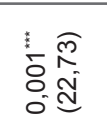 & \\
\hline & $\stackrel{\leftrightarrow}{\sim}$ & 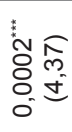 & $\begin{array}{l}8 \\
80 \\
0 \\
0 \\
0 \\
1\end{array}$ & & $\begin{array}{l}\dot{1} \\
\delta \\
\delta \\
0 \\
0 \\
0\end{array}$ & 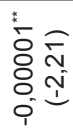 & $\begin{array}{l}8 \text { व } \\
\text { iv } \\
01\end{array}$ & $\begin{array}{l}\bar{*} \\
8 \\
0 \\
0\end{array}$ & $\begin{array}{l}\bar{\sigma} \\
\bar{\delta} \\
\overline{0} \\
\bar{\alpha}\end{array}$ & \\
\hline & $\stackrel{\mathscr{N}}{\sim}$ & 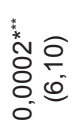 & $\begin{array}{l}8 \\
80 \\
0 \\
0 \\
\\
1\end{array}$ & & $\begin{array}{l}1 \\
\vdots \\
0 \\
0 \\
0 \\
0\end{array}$ & & $\begin{array}{l}8 \overline{1} \\
8 \stackrel{0}{0} \\
i=1\end{array}$ & $\begin{array}{l}-\widehat{0} \\
80 \\
0 \\
0 \\
0\end{array}$ & 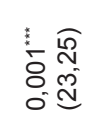 & \\
\hline & & $\begin{array}{l}\text { 山r } \\
\stackrel{\underline{r}}{0} \\
0 \\
0\end{array}$ & 岕 & ષ્ & $\begin{array}{l}\text { 山् } \\
\text { پ }\end{array}$ & o & $\stackrel{\rightleftarrows}{\mathrm{U}}$ & 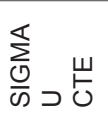 & 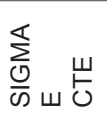 & \\
\hline
\end{tabular}


Los resultados del estudio de la relación no lineal muestran significancia de las variables dependientes PAFP y PAFP $^{2}$ en todas las regresiones, salvo en la estimación (26). Más específicamente, se encuentra que la variable PAFP está positivamente relacionada con el rendimiento de los dividendos, mientras que su forma cuadrática presenta una relación negativa. Dicha relación apunta a la idea de que un incremento en la propiedad de la AFP implica un aumento en el valor esperado condicional de la política de dividendos, esto hasta cerca de un $15,22 \%$ de propiedad (punto en el que se encuentra el máximo). Estos resultados son congruentes con los encontrados anteriormente por Ruiz et al. (2008) estudiando la relación para inversores institucionales como Bancos, pero se contradicen con sus resultados en relación a los Fondos de Inversión. En comparación con las regresiones (13)-(24), obtenidas por el modelo de Tobit, las cuales no incluyen el concepto de no linealidad de la relación estudiada, se observa que las variables ROE y ROA mantienen su relación positiva y significativa al 99\% para todas las regresiones en que son controladas. Esta similitud también es observada para las variables APAL1, APAL2, Q (se destaca que este aumenta su significancia a $95 \%$ ) y OPCRE. Esta no linealidad de la relación entre la propiedad institucional y la política de dividendos ha sido evidenciada y demostrada por Schooley y Barney (1994), Alangar et al. (1999), Farinha (2003), Gugler y Yurtoglu (2003), Correia da Silva et al. (2004), Farinha y López de Foronda (2005), Thomsen (2005) y Ruiz et al. (2008).

Con el fin de estudiar la robustez de los resultados obtenidos en el estudio de la relación cuadrática entre las variables estudiadas, se reestimaron las regresiones separando las variables independientes de las variables de control, buscando aislar el posible efecto resultante de la selección de las variables de control. Los resultados de este análisis se detallan en el cuadro 6. Este análisis de robustez muestra que la significancia y el signo de la relación para la variable dependiente PAFP y su forma cuadrática PAFP $^{2}$ se mantiene independiente de las variables de control que se incluyan en el modelo. Por otro lado, los resultados en cuanto a significancia y signo con respecto a las regresiones para el modelo Tobit considerando linealidad y no linealidad se mantienen para todas las variables de control.

Finalmente, el cuadro 7 presenta los resultados obtenidos para el modelo de Heckman de dos etapas, considerando las variables DIVYSI y PAFPSI como foco de estudio. Los resultados presentados evidencian la presencia de sesgo de selección para ambos modelos planteados (a) y (b), dado que el valor Mills es estadísticamente significativo para ambos casos. Esto implicaría que un aumento en los pagos de dividendos en un periodo determinado aumenta la probabilidad de que el nivel de propiedad de las AFP aumente para el periodo siguiente (lo que se respalda con las teorías que apuntan a que los mayores pagos de dividendos son recibidos como una señal positiva y con la hipótesis de preferencia institucional que implicaría que las AFP se comportan como un inversor prudente; también puede relacionarse con el pastoreo institucional, debido a que la señal percibida por una AFP que la lleve a invertir en una empresa puede conducir a otras AFP 


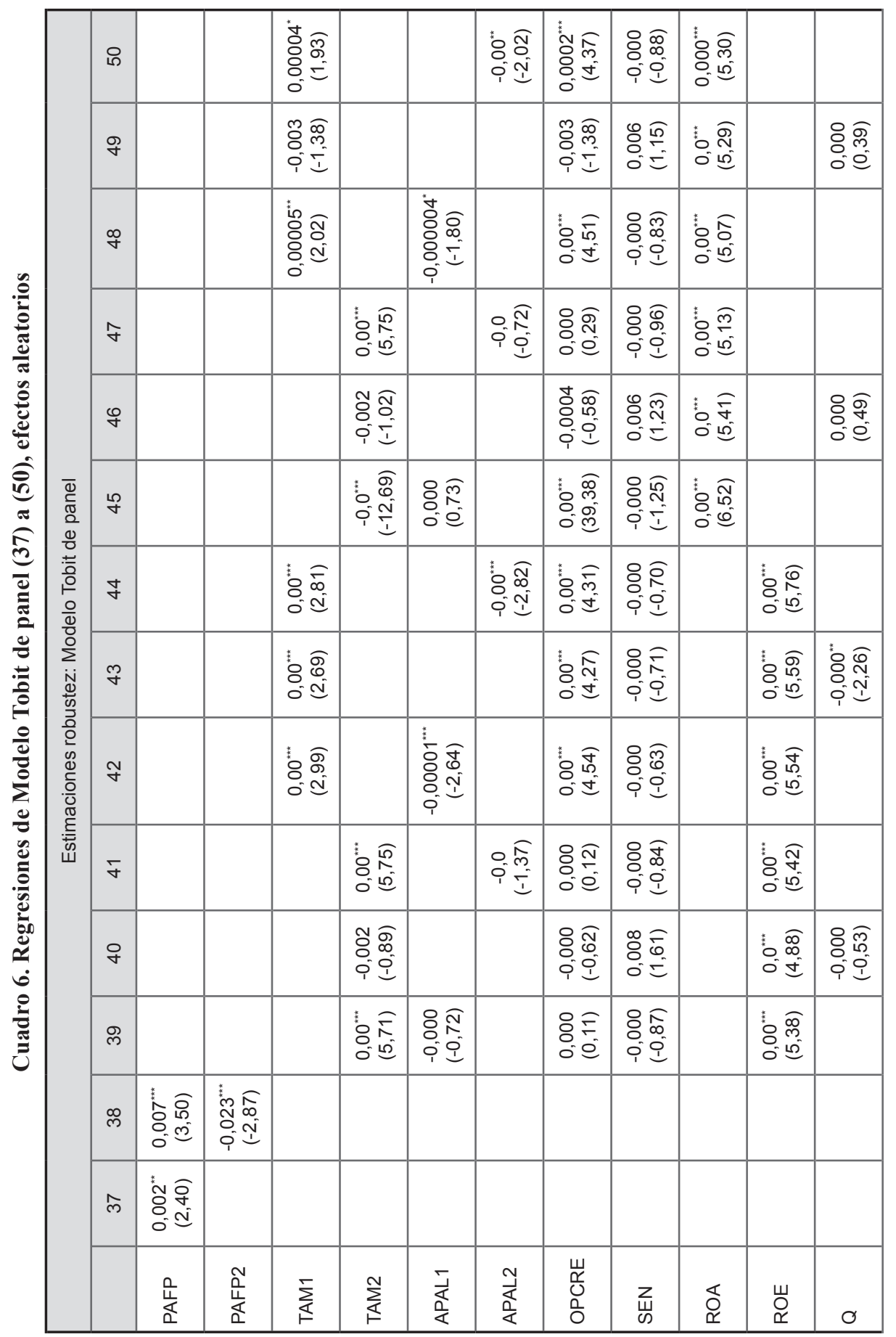




\begin{tabular}{|c|c|c|c|c|c|}
\hline \multirow{15}{*}{ 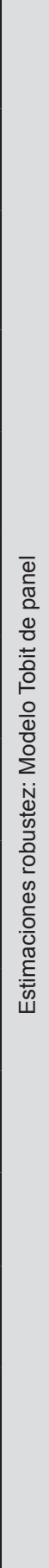 } & 오 & 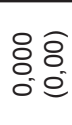 & $\begin{array}{l}1 \\
\overline{0} \\
0 \\
0 \\
0 \\
0\end{array}$ & 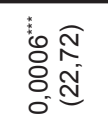 & \multirow[b]{15}{*}{ 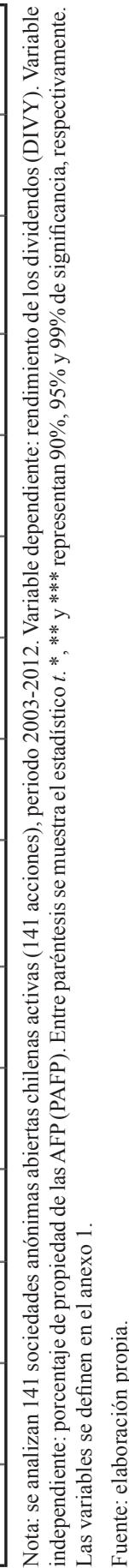 } \\
\hline & g & 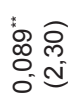 & 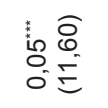 & 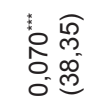 & \\
\hline & $\stackrel{\infty}{+}$ & $\begin{array}{l}8 . \overline{8} \\
0 \\
0\end{array}$ & $\begin{array}{l}1 \\
\overline{0} \\
8 \infty \\
0 \\
0\end{array}$ & 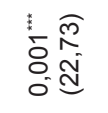 & \\
\hline & $\hat{f}$ & $\begin{array}{l}\text { E } \\
\text { : } \\
\text { i }\end{array}$ & $\begin{array}{l}\widehat{g} \\
0 \\
0 \\
0\end{array}$ & 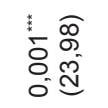 & \\
\hline & $\mathscr{q}$ & $\begin{array}{l}\text { No } \\
00 \\
0= \\
0\end{array}$ & 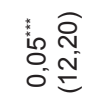 & 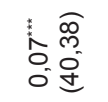 & \\
\hline & \&f & 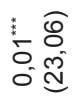 & 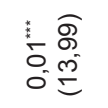 & 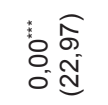 & \\
\hline & ষ & $\begin{array}{l}80 \\
80 \\
0.0 \\
0.1\end{array}$ & 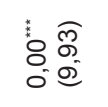 & 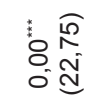 & \\
\hline & भ & 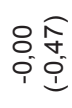 & 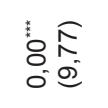 & : & \\
\hline & F & $\begin{array}{l}80 \\
80 \\
0 \\
0 \\
0 \\
0\end{array}$ & 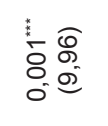 & $\begin{array}{l}\widehat{10} \\
0 \\
0 \\
0 \\
\mathbb{N}\end{array}$ & \\
\hline & ₹ & 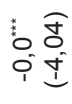 & $\begin{array}{l}0 \overline{5} \\
80 \\
0.0\end{array}$ & 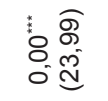 & \\
\hline & 우 & $\begin{array}{l}\text { 웅 } \\
0 \stackrel{0}{0}= \\
0=\end{array}$ & 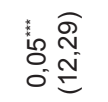 & $\begin{array}{c}0 \\
0 \\
0 \\
0 \\
0 \\
0 \\
0 \\
0\end{array}$ & \\
\hline & ల్ & 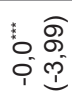 & $\begin{array}{l}\widehat{\widehat{N}} \\
8 \stackrel{0}{0.0} \\
0.0\end{array}$ & 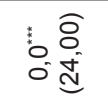 & \\
\hline & $\stackrel{\infty}{\infty}$ & 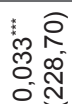 & 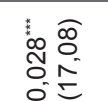 & $\begin{array}{l} \\
0 \\
0 \\
0 \\
0 \\
0 \\
0\end{array}$ & \\
\hline & $\hat{m}$ & 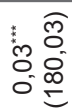 & 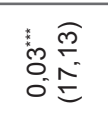 & $\begin{array}{l}\widehat{\sigma} \\
0 \\
0 \\
0\end{array}$ & \\
\hline & & 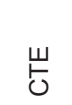 & 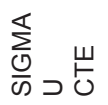 & 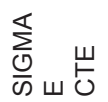 & \\
\hline
\end{tabular}


Cuadro 7. Método de Heckman en dos etapas

\begin{tabular}{|c|c|c|c|c|c|}
\hline \multicolumn{2}{|c|}{ V. dependiente } & \multirow{2}{*}{$\begin{array}{c}\text { AFPSI } \\
\text { (b) }\end{array}$} & \multicolumn{2}{|c|}{ V. dependiente } & \multirow{2}{*}{$\begin{array}{c}\text { DIVYSI } \\
\text { (b) }\end{array}$} \\
\hline & (a) & & & (a) & \\
\hline DIVY & $\begin{array}{c}2,046^{* * *} \\
(2,59)\end{array}$ & $\begin{array}{c}1,638^{* *} \\
(2,28)\end{array}$ & PAFP & $\begin{array}{l}0,892^{*} \\
(1,95)\end{array}$ & $\begin{array}{c}0,903^{* *} \\
(2,08)\end{array}$ \\
\hline Q & $\begin{array}{c}0,007^{* * *} \\
(2,81)\end{array}$ & & Q & $\begin{array}{l}-0,002 \\
(-0,79)\end{array}$ & \\
\hline SEN & $\begin{array}{l}-0,028 \\
(-0,30)\end{array}$ & $\begin{array}{l}-0,034 \\
(-0,35)\end{array}$ & ROES & $\begin{array}{c}0,008^{* * *} \\
(3,14)\end{array}$ & $\begin{array}{c}0,008^{* * *} \\
(3,26)\end{array}$ \\
\hline OPCRE & $\begin{array}{l}-0,038 \\
(-1,17)\end{array}$ & $\begin{array}{l}-0,026 \\
(-1,03)\end{array}$ & OPCRE & $\begin{array}{l}0,058^{*} \\
(1,79)\end{array}$ & $\begin{array}{l}0,055^{\star} \\
(1,91)\end{array}$ \\
\hline ROES & $\begin{array}{c}-0,006^{* *} \\
(-2,13)\end{array}$ & $\begin{array}{c}-0,006^{* *} \\
(-2,37)\end{array}$ & SEN & $\begin{array}{l}-0,015 \\
(-0,10)\end{array}$ & $\begin{array}{l}-0,035 \\
(-0,26)\end{array}$ \\
\hline TAM2 & $\begin{array}{c}0,324^{* * *} \\
(10,59)\end{array}$ & $\begin{array}{c}0,331^{* * *} \\
(11,43)\end{array}$ & TAM2 & $\begin{array}{c}-0,122^{*} \\
(-1,65)\end{array}$ & $\begin{array}{l}-0,125 \\
(-1,59)\end{array}$ \\
\hline APAL1 & & $\begin{array}{c}0,005^{* *} \\
(2,10)\end{array}$ & APAL1 & & $\begin{array}{l}-0,001 \\
(-0,51)\end{array}$ \\
\hline CTE & $\begin{array}{c}-5,914^{* * *} \\
(-10,26)\end{array}$ & $\begin{array}{c}-5,992^{* * *} \\
(-11,01)\end{array}$ & CTE & $\begin{array}{c}3,395^{* *} \\
(2,01)\end{array}$ & $\begin{array}{l}3,408^{*} \\
(1,92)\end{array}$ \\
\hline
\end{tabular}

Nota: modelos (a) y (b), valor Mills -1,126*** y -1,087*** respectivamente. Se consideran 141 sociedades anónimas activas en Chile para los años 2003-2012. El valor del parámetro es estadísticamente distinto a cero (estadístico de 0,00120). Entre paréntesis se muestra el estadístico $t$. ${ }^{*}, * *$ y *** representan $90 \%, 95 \%$ y $99 \%$ de significancia, respectivamente.

Fuente: elaboración propia.

a invertir en esta firma, aumentado su nivel de propiedad), apuntando a que las AFP escogen, al momento de invertir o no, a firmas con mayores pagos de dividendos, mayor tamaño, menor rentabilidad contable, mayores flujos de caja libre y mayores niveles de apalancamiento. Al ejercer su propiedad sobre la firma, hay mayor probabilidad de que la rentabilidad sobre los dividendos sea positiva, reafirmando los resultados obtenidos a lo largo de este documento sobre la incidencia de mayores niveles de propiedad, mayor rentabilidad contable, mayores oportunidades de crecimiento y menores tamaños sobre este resultado. Esto es consistente, además, con los resultados obtenidos al estimar las regresiones (1)-(12), los cuales revelaron la existencia de una relación positiva y significativa entre DIVY y PAFP. La relación positiva de la $\mathrm{Q}$ de Tobin (Q) y la variable dummy AFPSI sugiere que, en estos casos, un aumento en los flujos de caja libre durante el periodo anterior incentivan a las AFP a invertir en la empresa en el periodo siguiente. De manera similar, los resultados muestran que es posible que mayores oportunidades de crecimiento aumenten la probabilidad de tener una rentabilidad de los dividendos positiva. En definitiva, es posible que las empresas aumenten su nivel 
de pago buscando incentivar la inversión de estas entidades, lo que implica la existencia de un sesgo de selección.

\section{Conclusiones}

Este artículo examinó el impacto del nivel de propiedad institucional de las Administradoras de Fondos de Pensiones (AFP) sobre el pago de dividendos (representado como el rendimiento de los dividendos) de las empresas para el contexto chileno. Los resultados muestran en primer lugar la existencia de una relación lineal positiva y significativa entre el nivel de propiedad de las AFP y el pago de dividendos de las empresas en que invierten. Los resultados difieren a los encontrados por Azzam (2010), quien señala que la propiedad institucional tiene un impacto negativo en el pago de dividendos, y por Allen (2000), quien afirma que si en las empresas predominan los inversores bien informados se pagarán menos dividendos. Sin embargo, son respaldados por los expuestos por Short et al. (2002), Manos (2002), Almazán et al. (2005), Maury y Pajuste (2002), quienes también encuentran una relación positiva entre los pagos de dividendos y la propiedad de institucionales.

Se tuvo que tomar en cuenta el control de las observaciones censuradas, dado que en Chile, a pesar de la obligatoriedad de pago de mínimo un 30\% de las utilidades líquidas en forma de dividendos, existen empresas que no pagan dividendos para un periodo por no tener utilidades o por decidir realizar un pago de utilidades de años anteriores. Para el control de los casos sin dividendos, se utilizó un modelo Tobit de panel, cuyos resultados reconfirmaron la existencia de una relación lineal positiva entre las principales variables de estudio. También, se comprobó la existencia de una relación no lineal entre estas dos variables; específicamente, dicha relación es positiva para la variable PAFP y negativa para su forma cuadrática $\mathrm{PAFP}^{2}$.

Por último, se evidenció la existencia de sesgo de selección, revelándose que la decisión de invertir por parte de las AFP sobre una empresa en un periodo determinado se relaciona con el incremento en la política de dividendos del periodo anterior, sugiriendo que dicho aumento implica una mayor probabilidad de que la AFP decida invertir. Esta idea se ve respaldada con las teorías que apuntan a que los mayores pagos de dividendos son recibidos como una señal positiva por los inversores, con la hipótesis de preferencia institucional que implicaría que las AFP se comportan como un inversor prudente, por lo que estos captarán esta señal e invertirán en la empresa, y con la hipótesis de pastoreo institucional, lo que implicaría que las AFP al recibir la señal positiva se moverían en conjunto, aumentando el nivel de propiedad. Luego de ejercer su propiedad sobre la firma, los pagos de dividendos tienen más probabilidad de realizarse para el periodo siguiente con un aumento del nivel de propiedad. 


\section{Anexo 1. Descripción de las variables}

\begin{tabular}{|c|c|}
\hline Variable & Descripción \\
\hline DIVY & $\begin{array}{l}\text { Rendimiento del dividendo, ratio entre divi- } \\
\text { dendo por acción y su precio }\end{array}$ \\
\hline PAFP & $\begin{array}{l}\text { Cantidad de acciones en propiedad de las } \\
\text { AFP sobre el total de acciones emitidas }\end{array}$ \\
\hline TAM1 & Logaritmo natural de los ingresos totales \\
\hline TAM2 & $\begin{array}{l}\text { Logaritmo natural de la capitalización } \\
\text { bursátil }\end{array}$ \\
\hline APAL1 & $\begin{array}{l}\text { Deuda total sobre el valor libro de los ac- } \\
\text { tivos totales }\end{array}$ \\
\hline APAL2 & $\begin{array}{l}\text { Deuda total sobre el valor libro del patri- } \\
\text { monio }\end{array}$ \\
\hline SEN & $\begin{array}{l}\text { Diferencia entre la utilidad por acción en t y } \\
\text { la utilidad por acción en } \mathrm{t}-1 \text { sobre el precio } \\
\text { de la acción en t-1 }\end{array}$ \\
\hline Q & $\begin{array}{l}\text { Capitalización bursátil más pasivos sobre } \\
\text { los activos }\end{array}$ \\
\hline OPCRE & $\begin{array}{l}\text { Ratio entre valor de mercado y valor libro } \\
\text { del precio de la acción (P/VL) }\end{array}$ \\
\hline ROA & $\begin{array}{l}\text { Retorno sobre activos, ingreso neto sobre } \\
\text { total de activos }\end{array}$ \\
\hline ROE & $\begin{array}{l}\text { Retorno sobre patrimonio, ingreso neto so- } \\
\text { bre la propiedad de los accionistas }\end{array}$ \\
\hline
\end{tabular}

Fuente: elaboración propia.

\section{Referencias}

Agosin, M., and Pastén, E. (2003). Corporate governance in Chile. Documentos de Trabajo 209, Banco Central de Chile.

Alangar, S., Bathala, Ch. T., and Fao, R. P. (1999). The effect of institutional interest on the information content of dividend-change announcements. The Journal of Financial Research, 22 (4), 429-448.

Allen, F., Bernardo, A., and Welch, I. (2000). A theory of dividends based on tax clienteles. Journal of Finance, 55, 2499-2536.
Almazan, A., Hartzell, J. and Starks, L. (2005). Active institutional shareholders and costs of monitoring, evidence from executive compensation. Financial Management, 34 (4), 5-35.

Amihud, Y., and Murgia M. (1997). Dividends, taxes, and signaling: Evidence from Germany. The Journal of Finance, LII (1), 397-408.

Arrazola, M., De Hevia, J., y Mato, G. (1992). Determinantes de la distribución de dividendos. Investigaciones económicas, 16 (2), 235-258.

Azzam, I. (2010). The impact of institutional ownership and dividend policy on stock returns and volatility: Evidence from Egypt. International Journal of Business, 15 (4), 443-458.

Barclay, M. J., Smith, C. W., and Watts, R. L. (1995). The determinants of corporate leverage and dividend policies. Journal of Applied Corporate Finance, 7, 4-19.

Becht, M., and Röell, A. (1999). Blockholdings in Europe: An international comparison. European Economic Review, 43, 1049-1056.

Black, F. (1976). The dividend puzzle. Journal of Portfolio Management, 2, 5-8.

Chen, S., Ho, K., Lee C., and Shrestha, K. (2004). Nonlinear models in corporate finance research: Review, critique, and extensions. Review of Quantitative Finance and Accounting, 22 (2), 141-169.

Correia Da Silva, L., Georgen, M., and Renneboog, L. (2004). Dividend policy and corporate governance. Oxford University Press.

Faccio, L., and Lang, L. (2002). The ultimate ownership of western European corporations. Journal of Financial Economics, 65 (3), 365-395.

Faccio, L., Lang, L., and Young, L. (2001). Dividends and Expropriation. American Economic Review, 91 (1), 54-78.

Farinha, J. (2003). Dividend policy, corporate governance and the managerial entrenchment hypothesis: An empirical analysis. Journal of 
Business Finance and Accounting, 30 (9-10), 1173-1209.

Farinha, J., and López de Foronda, O. (2005). The relation between dividends and insider ownership in different legal systems: International evidence. Centro de Estúdios de economia industrial, do trabalho e da empresa.

Finchelstein, D. (2010). Different states, different internationalizations: An analysis of the process of firms. North Western University, Rio de Janeiro: LASA International Congress.

Finchelstein, D. (2012). Políticas públicas, disponibilidad de capital e internacionalización de empresas en América Latina: los casos de Argentina, Brasily Chile. Consejo Nacional de Investigaciones Científicas y Técnicas (Conicet), Argentina.

Fuentes, S. (2013). Inversiones de las AFP en empresas chilenas, determinantes y efectos en el mercado. Tesis para optar al grado de Magíster en Economía Aplicada, Universidad de Chile.

Fuenzalida, D., Nash, M., and Mongrut, S. (2008). Stocks splits la bolsa de valores de Lima ¿Afectan el rendimiento y la liquidez de los títulos? Estudios Gerenciales, 24 (109), 11-36.

Giner, E. y Salas, V. (1995). Explicaciones alternativas para la política de dividendos. Análisis empírico con datos empresariales españoles. Investigaciones Económicas, XIX (3), 329-348.

Grinstein, Y., and Michaely, R. (2005). Institutional holdings and payout policy. Journal of Finance, 60, 1389-1429.

Gugler, K., and Yurtoglu, B. (2003). Corporate governance and dividend pay-out policy in Germany. American Economic Review, 47 (4), 731-758.

Gutiérrez, M., Yáñez, M., and Umaña, B. (2012). Análisis de la política de pago de dividendos en empresas chilenas. Estudios Gerenciales, 28 (123), 27-42.
Han, K. C., Lee, S. H., and Suk, D. Y. (1999). Institutional Shareholders and Dividends. Journal of Financial and Strategic Decisions, 12 (1), 53-62.

Higgins, R. C. (1972). The corporate dividend - saving decisions. Journal of Financial and Quantitative Analysis, 7 (2), 1527-1541.

Higgins, R. C. (1981). Sustainable growth under inflation. Financial Management, 10, 36-40.

Howe, H., He, J., and Kao, W. (1992). One-time cash flow announcements and free cash flow theory: Share repurchases an special dividends. Journal of Finance, 47, 1963-1977.

Jensen, M. (1986). Agency costs of free cash flow, corporate finance, and take overs. The American Economic Review, 76 (2), 323-329.

La Porta, R., López de Silanes, F., and Shleifer, A. (1999). Corporate ownership around the world. The Journal of Finance, 54 (2), 471-517.

La Porta, R., López-de Silanes, F., Shleifer, A., and Vishny, R. (2000). Agency problem and dividend policies around the world. The Journal of Finance, 55 (1), 1-33.

Lefort, F. (2007). Board independence, firm performance and ownership concentration: Evidence from Chile. Journal of Business Research, 61, 615-622.

Lefort, F., and Walker, E. (2002). Cambios estructurales e integración. Discusión y análisis del mercado accionario chileno. Cuadernos de economía, 39 (116), 95-122.

Lin, J., Lee, Y., and Liu, Y. (2007). IPO Auctions and Private Information. Journal of Banking and Finance, 31, 1483-1500.

Mancinelli, L., and Ozkan, A. (2006). Ownership structure and dividend policy: Evidence from Italian firms. The European Journal of Finance, 12 (3), 265-282.

Manos, R. (2002). Dividend policy and agency theory: Evidence on Indian Firms. Institute for 
Development Policy and Management, Working Paper 41, Manchester University.

Maquieira, C. y Danús, M. (1998). Costos de agencia y costos de transacción, como determinantes de la tasa de pago de dividendos. Estudios de Administración, 5 (2), 49-77.

Maquieira, C. y Moncayo, I. (2004). Costos de agencia y costos de transacción como determinantes de la tasa de pago de dividendos: una extensión. Estudios de Administración, 11 (2), 1-33.

Maquieira, C. y Fuentes, O. (1997). Política de dividendos en Chile, 1993 y 1994. Estudios de Administración, 4 (1), 79-112.

Maury, C. B., and Pajuste, A. (2002). Controlling shareholders, agency problems, and dividend policy in Finland. Working Paper, Swedish School of Economics and Business Administration.

Miller, M., and Modigliani, F. (1961). A dividend policy, growth and the valuation of shares. Journal of Business, 34, 411-433.

Nash, M., and Fuenzalida, D. (2004). The effect of dividend distribution on share return in Chile. Estudios Gerenciales, 93, 99-113.

Pruitt, S. W., and Gitman, L. W. (1991). The interactions between the investment, financing, and dividend decisions of major US firms. Financial Review, 26 (33), 409-430.

Rozeff, M. (1982). Growth, beta and agency costs as determinants of dividend payout ratios. Journal of Financial Research, 5 (3), 249-259.
Rubin, A., and Smith, D. (2009). Institutional ownership, volatility and dividends. Journal of Banking and Finance, 35 (6), 1581-1597.

Ruiz, M., Santana, D., Aguilar, I., and Díaz, N. (2008). Propiedad institucional y dividendos. Revista Europea de Dirección y Economía de la Empresa, 17 (3), 93-110.

Schooley, D. K., and Barney, L. D. (1994). Using dividend policy and managerial ownership to reduce agency costs. Journal of Financial Research, 17 (3), 363-373.

Short, H., Zhang, H., and Keasey, K. (2002). The link between dividend policy and institutional ownership. Journal of Corporate Finance, 8, 105-122.

Sias, R. (2004). Institutional herding. Review of Financial Studies, 17, 165-206.

Smith, C. W., and Watts, R. L. (1992). The investment opportunity set and corporate financing, dividend, and compensation policies. Journal of financial Economics, 32 (3), 263-292.

Stein, R., Miranda, P., and Risco, R. (2011). Herding in Chile: The case of equity trading in the Chilean pension fund market. Estudios de Administración, 8 (1), 23-24.

Thomsen, S. (2005). Conflicts of interests aligned incentives? Blockholder ownership, dividends and firm value in the US and the EU. European Business Organization Law Review, 6 (2), 201-225. 